Preprint typeset in JHEP style - HYPER VERSION

hep-th/0304239

SNU-TP 03-009

\title{
Thermodynamic behavior of IIA string theory on a pp-wave
}

\author{
Seungjoon Hyun*, Jong-Dae Park ${ }^{\dagger \ddagger}$ and Sang-Heon Yi* \\ * Institute of Physics and Applied Physics, Yonsei University, Seoul 120-749, Korea \\ ${ }^{\dagger}$ Yonsei Visiting Research Center, Yonsei University, Seoul 120-749, Korea \\ ${ }^{\ddagger}$ Center for Theoretical Physics, Seoul National University, Seoul 151-742, Korea \\ E-mail : hyun@phya.yonsei.ac.kn, jdpark@phya.snu.ac.kr, \\ shyi@phya.yonsei.ac.kr
}

\begin{abstract}
We obtain the thermal one loop free energy and the Hagedorn temperature of IIA superstring theory on the pp-wave geometry which comes from the circle compactification of the maximally supersymmetric eleven dimensional one. We use both operator and path integral methods and find the complete agreement between them in the free energy expression. In particular, the free energy in the $\mu \rightarrow \infty$ limit is shown to be identical with that of IIB string theory on maximally supersymmetric pp-wave, which indicates the universal thermal behavior of strings in the large class of pp-wave backgrounds. We show that the zero point energy and the modular properties of the free energy are naturally incorporated into the path integral formalism.
\end{abstract}

KEYWORDS: Hagedorn temperature, Free energy, IIA string on pp-wave. 


\section{Contents}

1. Introduction and conclusion

2. Review: IIA superstring on the pp-wave geometry 3

3. Operator method 5

4. Free energy and Hagedorn temperature 7

5. Path integral approach 10

5.1 GS superstring at finite temperature

5.2 Complex scalar field with twisted boundary condition 13

5.3 Manifestly modular invariant one loop free energy

5.4 The modular invariance of vacuum amplitude and the flat space limit 16

6. Equivalence between operator and path integral methods 17

A. Heuristic derivation of the one loop free energy 19

B. Useful formulae 20

\section{Introduction and conclusion}

String/M theory is often considered as the theory of everything. In particular, it is expected to describe the early Universe [1]. In this sense, it is very important to understand the finite temperature physics of the string/M theory. As is well known, there are some difficulties in studying the thermodynamics of a theory which contains gravity, for example string theory. Gravity suffers from the Jeans' instability which is deeply related to the formation of black holes. Also, the energy of a system with gravity may be difficult to define. But, as was argued in [2], we may consider the weakly coupled low energy limit of such theory and get some insight on the thermodynamic behavior of the theory. The most notable feature in the finite temperature string theory is the existence of the Hagedorn temperature [3, 4] around the string scale due to the exponential growth of the level density of string states. This Hagedorn temperature is believed to be the signal of a phase transition [2], though there is no clear understanding on the physics above the Hagedorn temperature.

There were some interesting works [ [0, 6, 7, 8, 9, 10] on the finite temperature IIB superstring theory on the maximally supersymmetric ten-dimensional pp-wave spacetime [11] which can be obtained as the Penrose limit of the $A d S_{5} \times S^{5}$ spacetime and gives another 
solvable model for Green-Schwarz superstrings [12]. As one incarnation of AdS/CFT correspondence [13], the finite temperature behavior of IIB string theory on this pp-wave has been studied in connection with that of the dual $\mathcal{N}=4$ super Yang-Mills theory in the corresponding limit. In those works, one loop free energy is calculated and the dependency of the Hagedorn temperature on the RR-flux, $\mu$, is found. If we assume that the Hagedorn temperature of strings on the pp-wave geometry depends on $\mu$ smoothly so that it reduces to the flat space result in the $\mu \rightarrow 0$ limit, then, from the dimensional argument, it can be expanded in terms of dimensionless quantity $\sqrt{\alpha^{\prime}} \mu$ as

$$
T_{H}=\frac{1}{2 \pi \sqrt{2 \alpha^{\prime}}}\left(1+\sum_{n=1}^{\infty} a_{n}\left(\sqrt{\alpha^{\prime}} \mu\right)^{n}\right),
$$

where $a_{n}$ is the numerical constant depending on the details of pp-wave geometry. It was argued in [6] that this Hagedorn temperature in the case of IIB pp-wave geometry represents the limiting temperature of string theory where the free energy diverges. Soon after it was shown in [8] that the free energy does not diverge, but is singular, and hence the Hagedorn temperature represents the phase transition.

In this paper, we aim to study the thermodynamics of type IIA Green-Schwarz (GS) superstring on the ten dimensional pp-wave geometry [14],

$$
\begin{gathered}
d s^{2}=-2 d x^{+} d x^{-}-A\left(x^{I}\right) d x^{+} d x^{+}+\sum_{I=1}^{8} d x^{I} d x^{I}, \\
F_{+123}=\mu, \quad F_{+4}=-\frac{\mu}{3},
\end{gathered}
$$

where

$$
A=\left(\frac{\mu}{3}\right)^{2}\left(x_{1}^{2}+x_{2}^{2}+x_{3}^{2}+x_{4}^{2}\right)+\left(\frac{\mu}{6}\right)^{2}\left(x_{5}^{2}+x_{6}^{2}+x_{7}^{2}+x_{8}^{2}\right) .
$$

This geometry comes from the dimensional reduction of the maximally supersymmetric eleven dimensional pp-wave [15], and has one characteristic mass scale, $\mu$. In studying the thermal properties of IIA string theory on this pp-wave geometry, we use the canonical ensemble. The canonical ensemble may not be justified in the cases where the formation of the long string can not be ignored, as its fluctuations make the thermalization of the string gas difficult. In this case, the microcanonical ensemble should be used instead 16, [17, 18, 19, 20]. It would be interesting to study the thermodynamics of IIA string theory on pp-wave using the microcanonical ensemble and to see the implications.

There are two complementary approaches to calculate the one loop free energy of the finite temperature string theory. One is the operator method which can be adapted easily once the light-cone gauge is chosen. The other is the path integral approach which is conceptually more transparent and is ready to be generalized to higher loops. In this paper we compute the one loop free energy using both methods and find the perfect agreement. Though, on general grounds, this should be expected, the explicit confirmation is rather nontrivial. One issue in the path integral approach to compute thermal one loop partition function is to determine the correct path integral measure, which is settled down in this paper. 
We also obtain the Hagedorn temperature which takes the assumed form (1.1). It is found to be the monotonic function of $\sqrt{\alpha^{\prime}} \mu$ and goes to infinity as $\mu \rightarrow \infty$. In the same limit, the free energy is shown to become

$$
F=-\frac{L \pi}{6 \beta^{2}},
$$

which is exactly the same as that of IIB string theory on the pp-wave [10]. In general, the free energy of $d$ dimensional field theories at high temperature behaves as [2]

$$
F_{d} \sim V \beta^{-d}
$$

This suggests that in the large $\mu$ limit, the physics on the pp-wave geometry essentially reduces to two-dimensional one. It would be very interesting to pursue this line.

As will be shown later, the one loop free energy of IIA superstrings on the given ppwave geometry has very similar structure to the IIB counterpart, apart from the small modification due to the differences in the supersymmetry and their supermultiplets. This strongly suggests that the Hagedorn temperature signals the phase transition, like the IIB case [8].

Our main motivation for the present work is to understand the real degrees of freedom of IIA strings on pp-wave and eventually to reveal those of $\mathrm{M}$ theory on pp-wave. The eleven-dimensional maximally supersymmetric pp-wave can be obtained from the Penrose limit of $A d S_{4} \times S^{7}$ or $A d S_{7} \times S^{4}$. Since the M theory on those $A d S$ spacetimes are dual to three dimensional or six dimensional conformal field theories, respectively, it is natural to expect that, in the spirit of the AdS/CFT correspondence, the M theory on the pp-wave is dual to (the common sector of ) those theories in the corresponding limit. Our study on the finite temperature physics of IIA strings on pp-wave may give some clues to understand those dual theories.

This paper is organized as follows. In section two, we briefly review IIA string theory on the pp-wave geometry. In section three, we obtain the free energy of the weakly interacting string gas on the pp-wave using the operator method. In section four, we consider the asymptotics of the free energy and find the Hagedorn temperature which depends on the RR-flux through the difference of boson and fermion zero point energies. In section five, we use the path integral approach to calculate the one loop free energy. Using this approach, we clarify several issues, including the zero point energy and the modular properties of the one loop free energy. In section six, we show the complete agreement between the two results, one from the operator method and the other from the path integral method. We also give various comments including the correct thermal free energy of the GS strings on flat space. In the appendices, we give simple derivation of the one loop free energy using Coleman-Weinberg formula and also give some useful formulae used in the main text.

\section{Review: IIA superstring on the pp-wave geometry}

In this section we summarize some results for the type IIA superstring theory on the ten dimensional pp-wave spacetime, including the mode expansions, the normal ordered 
worldsheet Hamiltonian and momentum (see, for more details, 14, 21]). The IIA pp-wave geometry (1.2) admits 24 Killing spinors, as the torus compactification breaks 8 supersymmetries out of 32 in the eleven-dimensional pp-wave geometry. In the light-cone gauge, the worldsheet description of IIA Green-Schwarz superstrings on this pp-wave background is given by the two-dimensional, free massive $\mathcal{N}=(4,4)$ supersymmetric theory. The bosonic fields, the target space coordinates, split into two multiplets with masses proportional to $\frac{\mu}{3}$ and $\frac{\mu}{6}$ in the light-cone gauge. The fermionic superpartners, after kappa gauge fixing, also split into two multiplets with the same masses as bosonic partners, classified by the chirality of $S O(8)$ rotations in $x^{I}$ and $S O(4)$ rotations in $x^{i^{\prime} 1}$. This is due to the fact that eight Killing spinors, which correspond to the dynamical supersymmetry on the string worldsheet, do not explicitly depend on the coordinate $x^{+}$. Let us denote the chirality of $S O(8)$ and $\gamma^{1234}$ as superscripts 1,2 and subscripts \pm for fermions, $\psi_{ \pm}^{1,2}$, respectively. Then one can see that the theory contains two supermultiplets $\left(X^{i}, \psi_{-}^{1}, \psi_{+}^{2}\right)$ and $\left(X^{i^{\prime}}, \psi_{+}^{1}, \psi_{-}^{2}\right)$ whose masses are given by $\frac{m}{3}$ and $\frac{m}{6}$, respectively, with $m \equiv \mu \alpha^{\prime} p^{+}$.

The light-cone gauge fixed action for this IIA Green-Schwarz superstring becomes quadratic in its fields as

$$
\begin{gathered}
S_{L C}=-\frac{1}{4 \pi \alpha^{\prime}} \int d \sigma^{0} d \sigma^{1}\left[\partial_{\alpha} X^{I} \partial^{\alpha} X^{I}+\left(\frac{m}{3}\right)^{2}\left(X^{i}\right)^{2}+\left(\frac{m}{6}\right)^{2}\left(X^{i^{\prime}}\right)^{2}-i \psi_{-}^{1} \partial_{+} \psi_{-}^{1}-i \psi_{+}^{2} \partial_{-} \psi_{+}^{2}\right. \\
\left.-i \psi_{+}^{1} \partial_{+} \psi_{+}^{1}-i \psi_{-}^{2} \partial_{-} \psi_{-}^{2}+i \frac{2 m}{3} \psi_{+}^{2} \gamma^{4} \psi_{-}^{1}-i \frac{m}{3} \psi_{-}^{2} \gamma^{4} \psi_{+}^{1}\right]
\end{gathered}
$$

where $\alpha=0,1$ denotes worldsheet index and $\partial_{ \pm}=\partial_{0} \pm \partial_{1}$.

Each field in the multiplet $\left(X^{i}, \psi_{-}^{1}, \psi_{+}^{2}\right)$ can be mode expanded, in terms of frequencies

$$
n \geq 0 \quad ; \quad \omega_{n}=\sqrt{\left(\frac{m}{3}\right)^{2}+n^{2}}, \quad n<0 \quad ; \quad \omega_{n}=-\sqrt{\left(\frac{m}{3}\right)^{2}+n^{2}},
$$

as follows:

$$
\begin{aligned}
& X^{i}=i \sqrt{\frac{\alpha^{\prime}}{2}} \sqrt{\frac{1}{\omega_{0}}}\left(a^{i} e^{-i \omega_{0} \sigma^{0}}-a^{i \dagger} e^{i \omega_{0} \sigma^{0}}\right)+i \sqrt{\frac{\alpha^{\prime}}{2}} \sum_{n \neq 0}\left(\frac{1}{\omega_{n}} \alpha_{n}^{i} e^{-i n \sigma^{1}}+\frac{1}{\omega_{n}} \tilde{\alpha}_{n}^{i} e^{i n \sigma^{1}}\right) e^{-i \omega_{n} \sigma^{0}}, \\
& \psi_{-}^{1}=-i \sqrt{\frac{\alpha^{\prime}}{2}} \gamma^{4}\left(\chi e^{-i \omega_{0} \sigma^{0}}-\chi^{\dagger} e^{i \omega_{0} \sigma^{0}}\right)+\sum_{n \neq 0} c_{n}\left(\tilde{\psi}_{n} e^{i n \sigma^{1}}-i \frac{\omega_{n}-n}{\omega_{0}} \gamma^{4} \psi_{n} e^{-i n \sigma^{1}}\right) e^{-i \omega_{n} \sigma^{0}}, \\
& \psi_{+}^{2}=\sqrt{\frac{\alpha^{\prime}}{2}}\left(\chi e^{-i \omega_{0} \sigma^{0}}+\chi^{\dagger} e^{i \omega_{0} \sigma^{0}}\right)+\sum_{n \neq 0} c_{n}\left(\psi_{n} e^{-i n \sigma^{1}}+i \frac{\omega_{n}-n}{\omega_{0}} \gamma^{4} \tilde{\psi}_{n} e^{i n \sigma^{1}}\right) e^{-i \omega_{n} \sigma^{0}},
\end{aligned}
$$

where $c_{n}=\sqrt{\frac{\alpha^{\prime} \omega_{0}^{2}}{\omega_{0}^{2}+\left(\omega_{n}-n\right)^{2}}}$ is the normalization constant taken canonically. After the canonical quantization, the modes satisfy the following commutation relations,

$$
\left[a^{i}, a^{j \dagger}\right]=\delta^{i j}, \quad\left[\alpha_{n}^{i}, \alpha_{m}^{j}\right]=\omega_{n} \delta^{i j} \delta_{n+m, 0},
$$

\footnotetext{
${ }^{1}$ In what follows, the indices $I=1,2, \ldots, 8$ denote the 8 transverse coordinates, among which the indices $i=1, \ldots, 4$ denote the direction where RR field strengths are spanned and the indices $i^{\prime}=5, \ldots, 8$ denote the other directions. We also take the real and symmetric representation for $S O(8)$ gamma matrices.
} 


$$
\left\{\chi, \chi^{\dagger}\right\}=1, \quad\left\{\psi_{n}, \psi_{m}\right\}=\delta_{n+m, 0}, \quad\left\{\tilde{\psi}_{n}, \tilde{\psi}_{m}\right\}=\delta_{n+m, 0} .
$$

Each field in the multiplet $\left(X^{i^{\prime}}, \psi_{+}^{1}, \psi_{-}^{2}\right)$ can also be mode expanded and canonically quantized similarly with frequencies

$$
n \geq 0 \quad ; \quad \omega_{n}^{\prime}=\sqrt{\left(\frac{m}{6}\right)^{2}+n^{2}}, \quad n<0 \quad ; \quad \omega_{n}^{\prime}=-\sqrt{\left(\frac{m}{6}\right)^{2}+n^{2}} .
$$

Introducing the number operators $N_{i n}^{B}, N_{n}^{F}$ for the multiplet $\left(X^{i}, \psi_{-}^{1}, \psi_{+}^{2}\right)$, defined by

$$
\begin{array}{ccc}
n>0 & n=0 & n<0 \\
N_{i n}^{B}:=\frac{1}{\omega_{n}} \alpha_{-n}^{i} \alpha_{n}^{i}, & a^{i \dagger} a^{i}, & \frac{1}{\omega_{-n}} \tilde{\alpha}_{n}^{i} \tilde{\alpha}_{-n}^{i}, \\
N_{n}^{F}:=\psi_{-n} \psi_{n}, & \chi^{\dagger} \chi, & \tilde{\psi}_{n} \tilde{\psi}_{-n},
\end{array}
$$

and similarly $N_{i^{\prime} n}^{B}, N_{n}^{\prime F}$ for $\left(X^{i^{\prime}}, \psi_{+}^{1}, \psi_{-}^{2}\right)$, the worldsheet momentum and Hamiltonian can be written as

$$
\begin{gathered}
\mathcal{P}=N-\tilde{N}=\sum_{n=-\infty}^{\infty} n\left(\sum_{i=1}^{4} N_{i n}^{B}+\sum_{i^{\prime}=5}^{8} N_{i^{\prime} n}^{B}+N_{n}^{F}+N_{n}^{\prime F}\right), \\
\mathcal{H}=\alpha^{\prime} p^{+} p^{-}=\sum_{n=-\infty}^{\infty}\left[\left|\omega_{n}\right|\left(\sum_{i=1}^{4} N_{i n}^{B}+N_{n}^{F}\right)+\left|\omega_{n}^{\prime}\right|\left(\sum_{i^{\prime}=5}^{8} N_{i^{\prime} n}^{B}+N_{n}^{\prime F}\right)\right] .
\end{gathered}
$$

\section{Operator method}

In this section we use the operator method to study the thermodynamic properties of IIA strings on the pp-wave. We consider the canonical ensemble composed of an ideal gas of weakly interacting strings on the given background. We mainly focus on the thermodynamic partition function or the free energy of string gas, from which all the other thermodynamic quantities can be obtained.

The free energy of the string gas can be expressed in terms of the trace, $\operatorname{Tr}^{\prime}$, over the one-string physical states as

$$
\begin{aligned}
F & =\frac{1}{\beta} \operatorname{Tr}^{\prime}\left[(-1)^{\mathbf{F}} \ln \left(1-(-1)^{\mathbf{F}} e^{-\beta p^{0}}\right)\right] \\
& =-\sum_{l=1}^{\infty} \frac{1+(-1)^{l}}{2 l \beta} \operatorname{Tr}^{\prime}\left[(-1)^{\mathbf{F}} e^{-l \beta p^{0}}\right]-\sum_{l=1}^{\infty} \frac{1-(-1)^{l}}{2 l \beta} \operatorname{Tr}^{\prime} e^{-l \beta p^{0}},
\end{aligned}
$$

where $\mathbf{F}$ is the space-time fermion number operator ${ }^{2}$. The level matching condition $N=\tilde{N}$ can be implemented by introducing the Lagrange multiplier, $\tau_{1}$, as

$$
F=-\sum_{l=1}^{\infty} \frac{1+(-1)^{l}}{2 l \beta} \operatorname{Tr}\left[\int_{-1 / 2}^{1 / 2} d \tau_{1} e^{2 \pi i \tau_{1}(N-\tilde{N})}(-1)^{\mathbf{F}} e^{-l \beta p^{0}}\right]
$$

\footnotetext{
${ }^{2}$ One may use $a p^{+}+b p^{-}$instead of $\beta p^{0}$ for the Boltzman factor [6, 8, 10], which is connected with the grand canonical ensemble. The results for this substitution can be easily read from our results as the geometry is invariant under the boosting along the longitudinal direction with the rescaling of $\mu$.
} 


$$
-\sum_{l=1}^{\infty} \frac{1-(-1)^{l}}{2 l \beta} \operatorname{Tr}\left[\int_{-1 / 2}^{1 / 2} d \tau_{1} e^{2 \pi i \tau_{1}(N-\tilde{N})} e^{-l \beta p^{0}}\right]
$$

where $\operatorname{Tr}$ denotes the trace over the one-string Fock space states without the level matching condition.

In the light-cone formalism, which is particularly suitable for the given pp-wave geometry, the space-time energy $p^{0}=\frac{1}{\sqrt{2}}\left(p^{+}+p^{-}\right)$is split into the light-cone Hamiltonian, $p^{-}=\frac{1}{\alpha^{\prime} p^{+}} \mathcal{H}$, and kinematical momentum, $p^{+}$. In this formalism, the one-string states are represented by continuous $p^{+}$and the transverse oscillators. Thus the above $\operatorname{Tr}$ can be written as

$$
\operatorname{Tr}=\frac{L}{\sqrt{2} \pi} \int_{0}^{\infty} d p^{+} \operatorname{tr}_{\text {trans }},
$$

where $L$ is the (infinite) length of the longitudinal $\left(x^{9}=\frac{1}{\sqrt{2}}\left(x^{+}-x^{-}\right)\right)$direction and $\operatorname{tr}_{\text {trans }}$ denotes the trace for the transverse oscillators without level matching constraint.

The first term in the free energy expression in (3.2) is simply the integral over $p^{+}$of the Witten index:

$$
\operatorname{tr}_{\text {trans }}\left[\int_{-1 / 2}^{1 / 2} d \tau_{1} e^{2 \pi i \tau_{1}(N-\tilde{N})}(-1)^{\mathbf{F}} e^{-\frac{\beta}{\sqrt{2}} p^{-}}\right] .
$$

This Witten index, in our case, turns out to be unity, while it vanishes in the case of string theory on flat spacetime, due to fermion zero modes.

Let us define

$$
\tau_{2}:=\frac{1}{\sqrt{2}} \frac{l \beta}{2 \pi \alpha^{\prime} p^{+}},
$$

in terms of which the mass parameter, $m$, can be rewritten as

$$
m=\frac{l \beta \mu}{2 \sqrt{2} \pi \tau_{2}} .
$$

This change of variables leads to

$$
F=-\frac{L \pi}{24 \beta^{2}}-\sum_{l=1, \text { odd }}^{\infty} \frac{L}{4 \pi^{2} \alpha^{\prime}} \int_{0}^{\infty} \frac{d \tau_{2}}{\tau_{2}^{2}} e^{-\frac{l^{2} \beta^{2}}{4 \pi \alpha^{\prime} \tau_{2}}} \int_{-1 / 2}^{1 / 2} d \tau_{1} \operatorname{tr}_{\text {trans }}\left[e^{2 \pi i \tau_{1} \mathcal{P}} e^{-2 \pi \tau_{2} \mathcal{H}}\right]
$$

Note that $\mathcal{H}=\alpha^{\prime} p^{+} p^{-}$given in Eq. (2.6) has $l$-dependence through $m$.

The integrand, $\operatorname{tr}_{\text {trans }}\left[e^{2 \pi i \tau_{1} \mathcal{P}} e^{-2 \pi \tau_{2}} \mathcal{H}\right]$, can be easily computed using the following formulae

$$
\begin{aligned}
\prod_{i=1}^{4} \sum_{N_{i n}^{B}=0}^{\infty} e^{\left(-2 \pi \tau_{2}\left|\omega_{n}\right|+2 \pi i \tau_{1} n\right) N_{i n}^{B}}=\left(\frac{1}{1-e^{-2 \pi \tau_{2}\left|\omega_{n}\right|+2 \pi i \tau_{1} n}}\right)^{4}, \quad \text { for bosons } \\
\sum_{N_{n}^{F}=0}^{4}\left(\begin{array}{c}
4 \\
N_{n}^{F}
\end{array}\right) e^{\left(-2 \pi \tau_{2}\left|\omega_{n}\right|+2 \pi i \tau_{1} n\right) N_{n}^{F}}=\left(1+e^{-2 \pi \tau_{2}\left|\omega_{n}\right|+2 \pi i \tau_{1} n}\right)^{4}, \quad \text { for fermions },
\end{aligned}
$$


with similar ones for $N_{i^{\prime} n}^{B}, N_{n}^{\prime F}$. As a result, the free energy becomes

$$
\begin{aligned}
F=-\frac{\pi L}{24 \beta^{2}}- & \sum_{l=1, \text { odd }}^{\infty} \frac{L}{4 \pi^{2} \alpha^{\prime}} \int_{0}^{\infty} \frac{d \tau_{2}}{\tau_{2}^{2}} \int_{-1 / 2}^{1 / 2} d \tau_{1} e^{-\frac{l^{2} \beta^{2}}{4 \pi \alpha^{\prime} \tau_{2}}} \\
& \prod_{n=-\infty}^{\infty}\left(\frac{1+e^{-2 \pi \tau_{2}\left|\omega_{n}\right|+2 \pi i \tau_{1} n}}{1-e^{-2 \pi \tau_{2}\left|\omega_{n}\right|+2 \pi i \tau_{1} n}}\right)^{4}\left(\frac{1+e^{-2 \pi \tau_{2}\left|\omega_{n}^{\prime}\right|+2 \pi i \tau_{1} n}}{1-e^{-2 \pi \tau_{2}\left|\omega_{n}^{\prime}\right|+2 \pi i \tau_{1} n}}\right)^{4} .
\end{aligned}
$$

Since we have used the normal ordered Hamiltonian, the zero point energy contribution to the Hamiltonian cancels between bosons and fermions and does not appear in the above free energy expression.

\section{Free energy and Hagedorn temperature}

In this section, we obtain the Hagedorn temperature from the free energy given in the previous section. We will see that the Hagedorn temperature behaves smoothly with respect to RR-flux, $\mu$, and in fact is a monotonic function of $\mu$.

The Hagedorn temperature can be extracted from the asymptotic behavior of the free energy. In order to see its asymptotic behavior, it is convenient to introduce the following quantity:

$$
D_{b_{1}, b_{2}}\left(\tau_{1}, \tau_{2} ; m\right):=e^{2 \pi \tau_{2} \Delta_{b_{1}}(m)} \prod_{n=-\infty}^{\infty}\left(1-e^{-2 \pi \tau_{2} \sqrt{\left(n+b_{1}\right)^{2}+m^{2}}+2 \pi i \tau_{1}\left(n+b_{1}\right)-2 \pi i b_{2}}\right),
$$

where $\Delta_{b}(m)$ is defined by

$$
\Delta_{b}(m):=-\frac{m}{\pi} \sum_{p=1}^{\infty} \frac{\cos (2 \pi b p)}{p} K_{1}(2 \pi m p)=-\frac{1}{2 \pi^{2}} \sum_{p=1}^{\infty} \cos (2 \pi b p) \int_{0}^{\infty} d s e^{-p^{2} s-\frac{\pi^{2} m}{s}}
$$

with the modified Bessel function $K_{1}(x)$. The function $\Delta_{b}(m)$ can be identified as the physical zero point (or Casimir) energy for two dimensional massive boson or fermion field with the twisted boundary condition as will be shown in the path integral approach given in the next section. Note that

$$
\Delta_{b}(m) \stackrel{m \rightarrow 0}{\longrightarrow} \frac{1}{24}-\frac{1}{8}(2 b-1)^{2}, \quad \Delta_{b}(m) \stackrel{m \rightarrow \infty}{\longrightarrow} 0 .
$$

We can exhibit the free energy using $D_{a, b}\left(\tau_{1}, \tau_{2} ; m\right)$ as

$$
\begin{aligned}
F=-\frac{\pi L}{24 \beta^{2}}-\sum_{\substack{l=1 \\
\text { odd }}}^{\infty} \frac{L}{4 \pi^{2} \alpha^{\prime}} & \int_{0}^{\infty} \frac{d \tau_{2}}{\tau_{2}^{2}} \int_{-\frac{1}{2}}^{\frac{1}{2}} d \tau_{1} e^{-\frac{l^{2} \beta^{2}}{4 \pi \alpha^{\prime} \tau_{2}}} \\
\times & {\left[\frac{D_{0,1 / 2}\left(\tau_{1}, \tau_{2} ; \frac{m}{3}\right)}{D_{0,0}\left(\tau_{1}, \tau_{2} ; \frac{m}{3}\right)}\right]^{4}\left[\frac{D_{0,1 / 2}\left(\tau_{1}, \tau_{2} ; \frac{m}{6}\right)}{D_{0,0}\left(\tau_{1}, \tau_{2} ; \frac{m}{6}\right)}\right]^{4} . }
\end{aligned}
$$

Since $D_{0,1 / 2}\left(\tau_{1}, \tau_{2} ; m\right) / D_{0,0}\left(\tau_{1}, \tau_{2} ; m\right)$ diverges when $\tau_{1}$ and $\tau_{2}$ vanish, we consider the limits $\tau_{1}=0$ and $\tau_{2} \rightarrow 0$ for the integrand of the free energy to see the apparently 
divergent behavior of it [5, 6. This limit is sufficient to obtain the Hagedorn temperature, but it is subtle whether this limit captures the nature of the Hagedorn temperature (see the results from Brower, Lowe and Tan [8]). In the above limit, $m$ goes to infinity which make it difficult to extract the singular behavior of $D_{b_{1}, b_{2}}\left(\tau_{1}, \tau_{2} ; m\right)$. To avoid this difficulty, one can use the following modular property [22, 23]

$$
\left|D_{b_{1}, b_{2}}\left(\tau_{1}, \tau_{2} ; m\right)\right|^{2}=\left|D_{b_{2},-b_{1}}\left(-\frac{\tau_{1}}{|\tau|^{2}}, \frac{\tau_{2}}{|\tau|^{2}} ; m|\tau|\right)\right|^{2}=\left|D_{b_{1}, b_{2}+b_{1}}\left(\tau_{1}+1, \tau_{2} ; m\right)\right|^{2},
$$

with $\tau=\tau_{1}+i \tau_{2}$. In our cases $b_{1}, b_{2}=0,1 / 2$, it reduces to

$$
D_{b_{1}, b_{2}}\left(\tau_{1}, \tau_{2} ; m\right)=D_{b_{2},-b_{1}}\left(-\frac{\tau_{1}}{|\tau|^{2}}, \frac{\tau_{2}}{|\tau|^{2}} ; m|\tau|\right)=D_{b_{1}, b_{2}+b_{1}}\left(\tau_{1}+1, \tau_{2} ; m\right) .
$$

Under this transformation, the integrand in the free energy expression becomes

$$
\begin{aligned}
& {\left[\frac{D_{0,1 / 2}\left(\tau_{1}, \tau_{2} ; \frac{m}{3}\right)}{D_{0,0}\left(\tau_{1}, \tau_{2} ; \frac{m}{3}\right)}\right]^{4}\left[\frac{D_{0,1 / 2}\left(\tau_{1}, \tau_{2} ; \frac{m}{6}\right)}{D_{0,0}\left(\tau_{1}, \tau_{2} ; \frac{m}{6}\right)}\right]^{4} } \\
= & {\left[\frac{D_{1 / 2,0}\left(-\frac{\tau_{1}}{|\tau|^{2}}, \frac{\tau_{2}}{|\tau|^{2}} ; \frac{m}{3}|\tau|\right)}{D_{0,0}\left(-\frac{\tau_{1}}{|\tau|^{2}}, \frac{\tau_{2}}{|\tau|^{2}} ; \frac{m}{3}|\tau|\right)}\right]^{4}\left[\frac{D_{1 / 2,0}\left(-\frac{\tau_{1}}{|\tau|^{2}}, \frac{\tau_{2}}{|\tau|^{2}} ; \frac{m}{6}|\tau|\right)}{D_{0,0}\left(-\frac{\tau_{1}}{|\tau|^{2}}, \frac{\tau_{2}}{|\tau|^{2}} ; \frac{m}{6}|\tau|\right)}\right]^{4} . }
\end{aligned}
$$

In the limit $\tau_{1}=0, \tau_{2} \rightarrow 0, m|\tau|=l \beta \mu /(2 \sqrt{2} \pi)$ is finite and thus the leading term of the integrand is readily expressed as

$$
\exp \left[\frac{2 \pi}{\tau_{2}}\left\{4 \Delta_{1 / 2}\left(\frac{l \beta \mu}{6 \sqrt{2} \pi}\right)-4 \Delta_{0}\left(\frac{l \beta \mu}{6 \sqrt{2} \pi}\right)+4 \Delta_{1 / 2}\left(\frac{l \beta \mu}{12 \sqrt{2} \pi}\right)-4 \Delta_{0}\left(\frac{l \beta \mu}{12 \sqrt{2} \pi}\right)\right\}\right]
$$

The asymptotics of the free energy, then, reads as

$$
\begin{aligned}
F \sim & -\frac{L}{4 \pi^{2} \alpha^{\prime}} \sum_{l=1, \text { odd }}^{\infty} \int_{0}^{\infty} \frac{d \tau_{2}}{\tau_{2}^{2}} \exp \left[-\frac{l^{2} \beta^{2}}{4 \pi \alpha^{\prime} \tau_{2}}\right] \\
& \times \exp \left[\frac{2 \pi}{\tau_{2}}\left\{4 \Delta_{1 / 2}\left(\frac{l \beta \mu}{6 \sqrt{2} \pi}\right)-4 \Delta_{0}\left(\frac{l \beta \mu}{6 \sqrt{2} \pi}\right)+4 \Delta_{1 / 2}\left(\frac{l \beta \mu}{12 \sqrt{2} \pi}\right)-4 \Delta_{0}\left(\frac{l \beta \mu}{12 \sqrt{2} \pi}\right)\right\}\right] .
\end{aligned}
$$

The smallest temperature which gives rise to the singularity of the above free energy comes from the $l=1$ case, which is taken as the Hagedorn temperature $T_{H}=1 / \beta_{H}$ :

$$
\begin{aligned}
\frac{\beta_{H}^{2}}{8 \pi^{2} \alpha^{\prime}} & =4\left[\Delta_{1 / 2}\left(\frac{\beta_{H} \mu}{6 \sqrt{2} \pi}\right)-\Delta_{0}\left(\frac{\beta_{H} \mu}{6 \sqrt{2} \pi}\right)\right]+4\left[\Delta_{1 / 2}\left(\frac{\beta_{H} \mu}{12 \sqrt{2} \pi}\right)-\Delta_{0}\left(\frac{\beta_{H} \mu}{12 \sqrt{2} \pi}\right)\right] \\
& =\frac{\beta_{H} \mu}{3 \sqrt{2} \pi^{2}} \sum_{p=1}^{\infty} \frac{1-(-1)^{p}}{p}\left(2 K_{1}\left(\frac{\beta_{H} \mu}{3 \sqrt{2}} p\right)+K_{1}\left(\frac{\beta_{H} \mu}{6 \sqrt{2}} p\right)\right) .
\end{aligned}
$$

Hagedorn temperature is often defined as the temperature at which the thermal winding mode of the string becomes massless [24, 25, 2]. As will be shown in the next section, this gives the same result. It is interesting to observe that, if the effective tension of the thermal winding string, depending on the temperature, is defined as follows:

$$
\alpha_{e f f}^{\prime}(\beta \mu)=\left(4\left[\Delta_{1 / 2}\left(\frac{\beta \mu}{6 \sqrt{2} \pi}\right)-\Delta_{0}\left(\frac{\beta \mu}{6 \sqrt{2} \pi}\right)\right]+4\left[\Delta_{1 / 2}\left(\frac{\beta \mu}{12 \sqrt{2} \pi}\right)-\Delta_{0}\left(\frac{\beta \mu}{12 \sqrt{2} \pi}\right)\right]\right) \alpha^{\prime},
$$


then, the leading term $(l=1)$ in the free energy (4.7), after a suitable rescaling in $\tau_{2}$, can be written as

$$
F \sim-\frac{L}{4 \pi^{2} \alpha^{\prime}{ }_{e f f}} \int_{0}^{\infty} \frac{d \tau_{2}}{\tau_{2}^{2}} \exp \left[-\frac{\beta^{2}}{4 \pi \alpha^{\prime}{ }_{e f f} \tau_{2}}+\frac{2 \pi}{\tau_{2}}\right],
$$

and the Hagedorn temperature becomes

$$
\beta_{H}=2 \pi \sqrt{2 \alpha^{\prime}{ }_{\text {eff }}\left(\beta_{H} \mu\right)} .
$$

Note that these expressions on the free energy and the Hagedorn temperature take the same forms as those of the string theory on the flat spacetime and reduce to the flat spacetime case smoothly as $\mu$ goes to zero.

For the small value of $\mu$, the Hagedorn temperature can be expressed explicitly as a perturbative series in terms of $\sqrt{\alpha^{\prime}} \mu$. Since the series expansion for the zero point energy is given by (see the Appendix for the full expression and its derivation),

$$
\Delta_{1 / 2}(x)-\Delta_{0}(x)=\frac{x}{\pi} \sum_{p=1}^{\infty} \frac{1-(-1)^{p}}{p} K_{1}(2 \pi x p)=\frac{1}{8}-\frac{x}{2}+x^{2} \ln 2+\mathcal{O}\left(x^{3}\right),
$$

the Hagedorn temperature up to $\mu^{2}$ order is

$$
T_{H}=\frac{1}{2 \pi \sqrt{2 \alpha^{\prime}}}\left(1+\frac{1}{2} \sqrt{\alpha^{\prime}} \mu+\left(\frac{1}{8}-\frac{5}{18} \ln 2\right)\left(\sqrt{\alpha^{\prime}} \mu\right)^{2}\right) .
$$

On the other hand, the large $\mu$ asymptotics of the Hagedorn temperature can be obtained as

$$
\frac{\beta_{H}^{2}}{32 \pi^{2} \alpha^{\prime}} \sim \frac{1}{\pi} \sqrt{\frac{\beta_{H} \mu}{12 \sqrt{2} \pi}} \exp \left[-\frac{\beta_{H} \mu}{6 \sqrt{2}}\right] \stackrel{\mu \rightarrow \infty}{\longrightarrow} 0 ; T_{H} \stackrel{\mu \rightarrow \infty}{\longrightarrow} \infty,
$$

through the asymptotic expansion of the zero point energy :

$$
\Delta_{1 / 2}(x)-\Delta_{0}(x)=\frac{x}{\pi} \sum_{p=1}^{\infty} \frac{1-(-1)^{p}}{p} K_{1}(2 \pi x p) \sim \frac{\sqrt{x}}{\pi} e^{-2 \pi x} .
$$

The free energy in this limit becomes identical with the one in the IIB case [10]

$$
F=-\frac{L \pi}{6 \beta^{2}},
$$

which shows a kind of the universality in the $\mu \rightarrow \infty$ limit. Note that this is a typical free energy behavior of two dimensional field theories at high temperature [2]. It strongly indicates, at least in the weakly coupled regime, that strings on the pp-wave geometry, in the large $\mu$ limit, effectively live on the two-dimensional spacetime. The AdS/CFT correspondence suggests that the dual theory of the M theory on maximally supersymmetric pp-wave would be related to some common sector of six-dimensional $(2,0)$ theory or $\mathcal{N}=8$ three-dimensional conformal field theory. Since our IIA pp-wave geometry comes from this eleven-dimensional pp-wave, our IIA string theory should be deeply related to these dual theories. It would be interesting to see the implications for dual theories of the behavior of free energy in the large $\mu$ limit. 


\section{Path integral approach}

The path integral formalism is much better, compared to the operator approach, in obtaining the genus-one free energy expression with the manifest modular invariance. It also incorporates the zero point energy more naturally and is appropriate for extending to higher genera.

In this section, we use the path integral approach to obtain the thermal one loop partition function and the Hagedorn temperature. As will be shown in the next section, these agree perfectly with the results given by the operator method. We follow the standard formalism of thermal string theory and extend to GS superstring theory on pp-wave. In the following subsections, we present all the involved quantities in the path integral method and clarify various issues including the modular invariance and the zero point energy introduced in the operator method by hand.

\subsection{GS superstring at finite temperature}

In the finite temperature field theory, the time coordinate is Wick-rotated and compactified with the circumference $\beta$. Accordingly, the corresponding process in the finite temperature string theory is to euclideanize the target space coordinate, $X^{0}$, to $X_{E}^{0}$ with the compactification radius $\beta$. Along the compactified direction $X_{E}^{0}$, strings have the winding modes, denoted by $r$, as well as the momentum modes, denoted by $l$ and, thus, have the following periodicity conditions :

$$
\begin{gathered}
X_{E}^{0}\left(\sigma^{1}+2 \pi, \sigma^{2}\right)=X_{E}^{0}\left(\sigma^{1}, \sigma^{2}\right)+r \beta, \\
X_{E}^{0}\left(\sigma^{1}+2 \pi \tau_{1}, \sigma^{2}+2 \pi \tau_{2}\right)=X_{E}^{0}\left(\sigma^{1}, \sigma^{2}\right)+l \beta,
\end{gathered}
$$

where we also euclideanized worldsheet time as $\sigma^{2}=i \sigma^{0}$. The classical configurations which satisfy these boundary conditions are, up to constant term, given by

$$
X_{\text {wind }}\left(\sigma^{1}, \sigma^{2}\right)=\frac{r \beta}{2 \pi} \sigma^{1}+\frac{\left(l-r \tau_{1}\right) \beta}{2 \pi \tau_{2}} \sigma^{2} .
$$

Though the spacetime Wick rotation on the pp-wave geometry itself [26] is problematic, we can still perform the Wick rotation in the path integral of GS superstring on the pp-wave.

We start from the covariant IIA action 114] which comes from the double dimensional reduction of the kappa gauge fixed supermembrane actions on the eleven-dimensional ppwave and use the conformal gauge for the worldsheet metric. For convenience, we perform the field redefinitions in the fermions as

$$
S_{1}^{a}=-\left(\gamma^{4} \psi_{-}^{1}\right)^{a}, \quad S_{2}^{a}=\psi_{+}^{2}{ }^{a} \quad ; \quad S_{1}^{\prime a}=\left(\gamma^{4} \psi_{+}^{1}\right)^{a}, \quad S_{2}^{\prime a}=\psi_{-}^{2 a} .
$$

Then the action becomes

$$
S_{E}=\frac{1}{8 \pi \alpha^{\prime}} \int d z d \bar{z}\left[-4\left(\partial X^{+} \bar{\partial} X^{-}+\bar{\partial} X^{+} \partial X^{-}\right)+4 \partial X^{I} \bar{\partial} X^{I}-4 A\left(X^{I}\right) \partial X^{+} \bar{\partial} X^{+}\right.
$$




$$
\begin{gathered}
+8 i\left\{\partial X^{+}\left(S_{1} \bar{\partial} S_{1}+S_{1}^{\prime} \bar{\partial} S_{1}^{\prime}\right)+\bar{\partial} X^{+}\left(S_{2} \partial S_{2}+S_{2}^{\prime} \partial S_{2}^{\prime}\right)\right. \\
\left.\left.-\left(\frac{\mu}{3}\right) \partial X^{+} \bar{\partial} X^{+}\left(2 S_{2} S_{1}+S_{2}^{\prime} S_{1}^{\prime}\right)\right\}\right]
\end{gathered}
$$

where $z=\sigma^{1}+i \sigma^{2}$ and $\bar{z}=\sigma^{1}-i \sigma^{2}$ with $d z d \bar{z}=2 d \sigma^{1} d \sigma^{2}$. For the calculation of the one loop amplitude via the path integral approach in the Green-Schwarz(GS) superstring, we should take into account the boundary conditions for the GS fermions, $S^{a}$. They are spacetime fermions and, thus, should be antiperiodic along the thermal direction $X_{E}^{0}$ in the finite temperature formalism. Therefore the boundary conditions for the fermions, consistent with the periodicity conditions (5.1), are 27]

$$
S_{1}^{a}(z+2 \pi)=(-1)^{r} S_{1}^{a}(z), \quad S_{1}^{a}(z+2 \pi \tau)=(-1)^{l} S_{1}^{a}(z),
$$

with the same conditions for $S_{1}^{\prime}(z)$ and similar ones for $S_{2}(\bar{z}), S_{2}^{\prime}(\bar{z})$ under $\bar{z}$ periodicity.

The one loop free energy can be represented as a path integral over the gauge fixed action as

$$
\begin{gathered}
-\beta F=Z_{T^{2}}(\beta)=\int_{\mathcal{F}} \frac{d \tau_{1} d \tau_{2}}{2 \tau_{2}} \sum_{r, l \in \mathbf{Z}} \int_{T^{2} /(r, l)} \mathcal{D} X^{+} \mathcal{D} X^{-} \mathcal{D} b \mathcal{D} c \mathcal{D} X^{I} \mathcal{D} S_{1,2} \mathcal{D} S_{1,2}^{\prime} \\
\times e^{-S_{E}-S_{g h}}\left(\operatorname{det} \partial X^{+}\right)^{-4}\left(\operatorname{det} \bar{\partial} X^{+}\right)^{-4}
\end{gathered}
$$

where $(r, l)$ denotes the boundary conditions Eq. (5.5) for the fermions $S_{1,2}^{a}, S_{1,2}^{\prime a}$ on the torus, and $S_{g h}$ is the same ghost action as in the flat space case. $\mathcal{F}$ denotes the fundamental region of the torus

$$
\mathcal{F}:=\left\{\left(\tau_{1}, \tau_{2}\right) ; \quad-\frac{1}{2}<\tau_{1} \leq \frac{1}{2}, \quad \tau_{2}>0, \quad|\tau| \geq 1\right\} .
$$

Note that the determinant in the above expression comes from the $\kappa$ symmetry gauge fixing ${ }^{3}$.

We perform, firstly, the integration over the longitudinal directions $X^{ \pm}$. As usual, we separate the fields into the classical part and the quantum fluctuation one as

$$
X^{ \pm}(z, \bar{z})=X_{c l}^{ \pm}+\delta X^{ \pm}, \quad X_{c l}^{ \pm}=x_{0}^{ \pm}+\frac{-i}{\sqrt{2}} X_{\text {wind }}
$$

The classical thermal winding configuration, $X_{c l}^{ \pm}$, leads to the action value

$$
S_{E}\left[X_{c l}^{ \pm}\right]=\frac{\beta^{2}|r \tau-l|^{2}}{4 \pi \alpha^{\prime} \tau_{2}}=: S_{\beta}(r, l)
$$

and the path integrals over $X^{ \pm}$are given by those of $\delta X^{ \pm}$around this configuration.

The computation of the path integral for the one loop free energy can be performed analogously to that of the one loop vacuum amplitude in the GS superstrings on the flat

\footnotetext{
${ }^{3}$ It has been argued [27] that it is not easy to incorporate $\kappa$ symmetry in the path integral of GS superstring rigorously. Nevertheless, the path integral defined as (5.6) will be shown to be consistent with the operator formalism.
} 
spacetime [28] and on the IIB pp-wave [29]. First, we integrate over $\delta X^{-}$which leads to the delta functional $\delta\left[\partial \bar{\partial} \delta X^{+}\right]=\delta\left[\delta X^{+}\right] / \operatorname{det}(\partial \bar{\partial})$. The integration over $\delta X^{+}$with this delta functional restricts $\delta X^{+}$to be zero in the integrand and, as usual, $1 / \operatorname{det}(\partial \bar{\partial})$ term cancels out with the $b c$ ghost contribution except for the zero mode, $x_{0}^{ \pm}$, contribution. The path integrals over the zero modes, $x_{0}^{ \pm}$, give rise to $\beta L /\left(4 \pi^{2} \alpha^{\prime} \tau_{2}\right)$ where $L$ is the (infinite) length of the longitudinal direction used in the operator method given earlier. Therefore the path integrals over $X^{ \pm}$and Faddeev-Popov ghosts around the classical configurations (5.2) give

$$
\sum_{r, l \in \mathbf{Z}} \frac{\beta L}{4 \pi^{2} \alpha^{\prime} \tau_{2}} e^{-S_{\beta}(r, l)},
$$

which is the same as the one given in [1, 2, 30]. In addition, $X^{ \pm}$fields in the residual integrand are replaced with $X_{c l}^{ \pm}$.

After the field independent rescaling of fermions, $S_{1,2}, S_{1,2}^{\prime}$, whose Jacobian cancels out the determinant factor $\operatorname{det}\left(\bar{\partial} X_{c l}^{+}\right) \operatorname{det}\left(\partial X_{c l}^{+}\right)$, the one loop free energy becomes the path integral over the transverse fields with the Euclidean action:

$$
\begin{aligned}
S^{\prime E}\left[X^{I}, S\right]= & \frac{1}{8 \pi \alpha^{\prime}} \int d z d \bar{z}\left[4 \partial X^{I} \bar{\partial} X^{I}+\left(\frac{m^{\prime}}{3}\right)^{2}\left(X^{i}\right)^{2}+\left(\frac{m^{\prime}}{6}\right)^{2}\left(X^{i^{\prime}}\right)^{2}\right. \\
& \left.+S_{1} \bar{\partial} S_{1}+S_{1}^{\prime} \bar{\partial} S_{1}^{\prime}-S_{2} \partial S_{2}-S_{2}^{\prime} \partial S_{2}^{\prime}+\left(\frac{m^{\prime}}{3}\right) S_{2} S_{1}+\left(\frac{m^{\prime}}{6}\right) S_{2}^{\prime} S_{1}^{\prime}\right]
\end{aligned}
$$

where

$$
m^{\prime}:=\frac{\mu \beta}{2 \sqrt{2} \pi \tau_{2}}|r \tau-l|
$$

As a result, the one loop free energy for a closed string gas can be written as

$$
-\beta F=Z_{T^{2}}(\beta)=\int_{\mathcal{F}} \frac{d \tau_{1} d \tau_{2}}{2 \tau_{2}} \sum_{r, l \in \mathbf{Z}} \frac{\beta L}{4 \pi^{2} \alpha^{\prime} \tau_{2}} e^{-S_{\beta}(r, l)} Z_{\text {trans }}^{(r, l)}\left(\tau_{1}, \tau_{2}\right),
$$

where $Z_{\text {trans }}^{(r, l)}\left(\tau_{1}, \tau_{2}\right)$ denotes the path integrals over the transverse fields:

$$
Z_{\text {trans }}^{(r, l)}\left(\tau_{1}, \tau_{2}\right):=\int_{T^{2} /(r, l)} \mathcal{D} X^{I} \mathcal{D} S_{1,2}^{a} e^{-S^{\prime E}\left[X^{I}, S\right]} .
$$

Note that $(r, l)=(0,0)$ part of the above free energy expression corresponds to the one loop vacuum amplitude, which is zero in our path integral formulation in parallel with the type IIB case [29]. We will give more comments on this vacuum amplitude later.

To get the transverse partition function (5.14), it is convenient [31, 32] to use the new coordinates, $\xi^{1}, \xi^{2}$, defined by $z=\xi^{1}+\tau \xi^{2}, \bar{z}=\xi^{1}+\bar{\tau} \xi^{2}$, which give $d z d \bar{z}=d^{2} \xi 2 \tau_{2}$ and $\partial \bar{\partial}=\left(1 / 2 \tau_{2}\right)^{2}\left[|\tau|^{2} \partial_{1}^{2}+\partial_{2}^{2}-2 \tau_{1} \partial_{1} \partial_{2}\right]$. The various fields $X^{i}, X^{i^{\prime}}, S_{1,2}^{a}, S_{1,2}^{\prime a}$ on the torus are Fourier expanded as

$$
\begin{aligned}
X\left(\xi_{1}, \xi_{2}\right) & =\sum_{n_{1}, n_{2}} x_{n_{1}, n_{2}} e^{i n_{1} \xi_{1}+i n_{2} \xi_{2}} \\
S\left(\xi_{1}, \xi_{2}\right) & =\sum_{n_{1}, n_{2}} S_{n_{1}, n_{2}} \exp \left[i\left(n_{1}+\frac{1-(-1)^{r}}{4}\right) \xi_{1}+i\left(n_{2}+\frac{1-(-1)^{l}}{4}\right) \xi_{2}\right],
\end{aligned}
$$


and the eigenvalues of those fields are given by

$$
\begin{aligned}
& \left(-4 \partial \bar{\partial}+M^{2}\right) X=E_{n_{1}, n_{2}} X ; \quad E_{n_{1}, n_{2}}=\left(\frac{1}{\tau_{2}}\right)^{2}\left|n_{1} \tau-n_{2}\right|^{2}+M^{2}, \\
& \left(-4 \partial \bar{\partial}+M^{2}\right) S=E_{n_{1}, n_{2}}^{(r, l)} S ; \quad E_{n_{1}, n_{2}}^{(r, l)}=\left(\frac{1}{\tau_{2}}\right)^{2}\left|\left(n_{1}+\frac{1-(-1)^{r}}{4}\right) \tau-\left(n_{2}+\frac{1-(-1)^{l}}{4}\right)\right|^{2}+M^{2},
\end{aligned}
$$

where $M$ denotes $\frac{m^{\prime}}{3}$ or $\frac{m^{\prime}}{6}$.

\subsection{Complex scalar field with twisted boundary condition}

The above type of path integrals was calculated in [33]. In this subsection we present the calculation in our context and clarify several points. Since the generalization to other cases are straightforward, it will be sufficient to consider the path integral of a massive complex bosonic field $\phi$ of mass $M$ with the twisted boundary conditions $\phi\left(\xi_{1}+2 \pi, \xi_{2}\right)=$ $e^{2 \pi i b_{1}} \phi\left(\xi_{1}, \xi_{2}\right)$ and $\phi\left(\xi_{1}, \xi_{2}+2 \pi\right)=e^{2 \pi i b_{2}} \phi\left(\xi_{1}, \xi_{2}\right)$. The energy eigenvalues with these boundary conditions are given by

$$
E_{n_{1}, n_{2}}^{\left(b_{1}, b_{2}\right)}=\left(\frac{1}{\tau_{2}}\right)^{2}\left|\left(n_{1}+b_{1}\right) \tau-\left(n_{2}+b_{2}\right)\right|^{2}+M^{2},
$$

and the partition function becomes

$$
Z_{\phi}(M):=\int \mathcal{D} \phi \mathcal{D} \bar{\phi} e^{-\int d^{2} \xi \tau_{2} \bar{\phi}\left(-4 \partial \bar{\partial}+M^{2}\right) \phi}=\prod_{n_{1}, n_{2}}\left(\tau_{2} E_{n_{1}, n_{2}}^{\left(b_{1}, b_{2}\right)}\right)^{-1}
$$

where we have absorbed the overall constant in the action by the field redefinition of $\phi$. As usual, this determinant can be regulated and evaluated using the $\zeta$-function method by considering

$$
G(s):=\sum_{n_{1}, n_{2} \in \mathbf{Z}}\left(\frac{\mu_{R}^{2}}{\tau_{2} E_{n_{1}, n_{2}}^{\left(b_{1}, b_{2}\right)}}\right)^{s}, \quad \operatorname{Re} s>1,
$$

where we have introduced the renormalization scale $\mu_{R}$ to insure the function $G(s)$ dimensionless in the $\zeta$-function scheme. As is well-known, this corresponds to the introduction of the cosmological constant term in the string worldsheet action as a counter term. Note also that we have included the factor $\tau_{2}$ which comes from the Lagrangian measure of the coordinate transformation from $(z, \bar{z})$ to $\left(\xi^{1}, \xi^{2}\right)$. As was pointed out in [33], it is required to perform the ultraviolet renormalization in the above determinant expression ${ }^{4}$, and our insertion of the renormalization scale, $\mu_{R}$, takes care of that. By the analytic continuation of $s$, the renormalized determinant can be evaluated through

$$
e^{G^{\prime}(0)}=\prod_{n_{1}, n_{2}} \frac{\mu_{R}^{2}}{\tau_{2} E_{n_{1}, n_{2}}^{\left(b_{1}, b_{2}\right)}}, \quad G^{\prime}(s):=\frac{d}{d s} G(s) .
$$

\footnotetext{
${ }^{4}$ In 33], "specific heat" is treated as the quantity for the renormalization prescription.
} 
It is sufficient to consider $G(s)$ up to the linear order in $s$ to get $G^{\prime}(0)$. Denoting $a=$ $\left(n_{1}+b_{1}\right) \tau_{1}-b_{2}$ and $c=\tau_{2} \sqrt{\left(n_{1}+b_{1}\right)^{2}+M^{2}}$, we get

$$
\begin{aligned}
G(s) & =\sum_{n_{1}, n_{2} \in \mathbf{Z}} \frac{\left(\mu_{R}^{2} \tau_{2}\right)^{s}}{\left[\left(n_{2}+a\right)^{2}+c^{2}\right]^{s}} \\
& =\left(\mu_{R}^{2} \tau_{2}\right)^{s} \sum_{n_{1} \in \mathbf{Z}}\left[\sqrt{\pi} c^{1-2 s} \frac{\Gamma\left(s-\frac{1}{2}\right)}{\Gamma(s)}+\frac{4 \pi^{s} c^{\frac{1}{2}-s}}{\Gamma(s)} \sum_{p=1}^{\infty} \frac{\cos (2 \pi a p)}{p^{\frac{1}{2}-s}} K_{\frac{1}{2}-s}(2 \pi c p)\right],
\end{aligned}
$$

where we have used the formula in the Appendix, (B.2), for $n_{2}$ summation. Using the formula (B.2) once again, we can see that the first term in the sum can be expanded in terms of $s$ as

$$
\begin{aligned}
\frac{\Gamma\left(s-\frac{1}{2}\right)}{\Gamma(s)} \sum_{n_{1}} \frac{\sqrt{\pi}}{c^{2 s-1}} & =\pi \frac{\Gamma(s-1)}{\Gamma(s)} \tau_{2}^{1-2 s} M^{2-2 s}+\frac{4 \pi^{s} \tau_{2}^{1-2 s} M^{1-s}}{\Gamma(s)} \sum_{p=1}^{\infty} \frac{\cos \left(2 \pi b_{1} p\right)}{p^{1-s}} K_{1-s}(2 \pi M p) \\
& =\pi \tau_{2} M^{2}+s\left[\pi \tau_{2} M^{2}\left(-1+\ln M^{2} \tau_{2}^{2}\right)-4 \pi \tau_{2} \Delta_{b_{1}}(M)\right]+\mathcal{O}\left(s^{2}\right),
\end{aligned}
$$

where $\Delta_{b}(m)$, the function defined by (4.2), appears naturally in this approach. The second term inside the sum is also expanded as

$$
\frac{4 \pi^{s} c^{\frac{1}{2}-s}}{\Gamma(s)} \sum_{p=1}^{\infty} \frac{\cos (2 \pi a p)}{p^{\frac{1}{2}-s}} K_{\frac{1}{2}-s}(2 \pi c p)=s\left[4 c^{\frac{1}{2}} \sum_{p=1}^{\infty} \frac{\cos (2 \pi a p)}{p^{\frac{1}{2}}} K_{\frac{1}{2}}(2 \pi c p)\right]+\mathcal{O}\left(s^{2}\right) .
$$

To proceed, note that $K_{\frac{1}{2}}(z)=\sqrt{\frac{\pi}{2 z}} e^{-z}$ and

$$
2 \sum_{p=1}^{\infty} \frac{\cos (2 \pi a p)}{p} e^{-2 \pi c p}=\sum_{p=1}^{\infty} \frac{1}{p}\left(q^{p}+\bar{q}^{p}\right)=-2|\ln (1-q)|,
$$

where $q=e^{2 \pi i a-2 \pi c}=e^{2 \pi i\left(n_{1}+b_{1}\right) \tau_{1}-2 \pi \tau_{2} \sqrt{\left(n_{1}+b_{1}\right)^{2}+M^{2}}-2 \pi i b_{2}}$. Finally, with $\left(\mu_{R}^{2} \tau_{2}\right)^{s}=1+$ $s \ln \left(\mu_{R}^{2} \tau_{2}\right)+\mathcal{O}\left(s^{2}\right)$, we obtain

$$
\begin{aligned}
G^{\prime}(0)=- & \pi \tau_{2} M^{2}+\pi \tau_{2} M^{2} \ln \left(\frac{M^{2} \tau_{2}}{\mu_{R}^{2}}\right)-4 \pi \tau_{2} \Delta_{b_{1}}(M) \\
& -2 \ln \left|\prod_{n_{1}=-\infty}^{\infty}\left(1-e^{2 \pi i\left(n_{1}+b_{1}\right) \tau_{1}-2 \pi \tau_{2} \sqrt{\left(n_{1}+b_{1}\right)^{2}+M^{2}}-2 \pi i b_{2}}\right)\right| .
\end{aligned}
$$

In all, the partition function for the massive complex scalar field $\phi$ is given by

$$
Z_{\phi}(M)=\left[e^{2 \pi \tau_{2} \Delta_{b_{1}}^{R}(M)}\left|\prod_{n_{1}}\left(1-e^{2 \pi i\left(n_{1}+b_{1}\right) \tau_{1}-2 \pi \tau_{2} \sqrt{\left(n_{1}+b_{1}\right)^{2}+M^{2}}-2 \pi i b_{2}}\right)\right|\right]^{-2},
$$

where

$$
\Delta_{b_{1}}^{R}(M)=\Delta_{b_{1}}(M)-\frac{1}{4} M^{2}\left[\ln \frac{M^{2} \tau_{2}}{\mu_{R}^{2}}-1\right]
$$


The above $\Delta_{b_{1}}^{R}(M)$ is the so-called zero point energy (or Casimir energy) on the worldsheet. But we emphasize that this is the renormalized quantity, not the physical one in the context of the renormalizable quantum field theory [34]. To get the physical quantity we need to impose the renormalization condition. We will take it by the criterion

$$
\Delta_{b}^{p h}(M) \longrightarrow 0, \quad \text { when } \quad M \longrightarrow \infty,
$$

which is the standard choice in the Casimir energy literature [35] (see also [36, 37]). This consideration finally gives us the physical quantity $\Delta_{b_{1}}^{p h}(M)=\Delta_{b_{1}}(M)$, and it will be economical to write the partition function only with the physical zero point energy as

$$
Z_{\phi}(M)=\left[e^{2 \pi \tau_{2} \Delta_{b_{1}}(M)}\left|\prod_{n_{1}}\left(1-e^{2 \pi i\left(n_{1}+b_{1}\right) \tau_{1}-2 \pi \tau_{2} \sqrt{\left(n_{1}+b_{1}\right)^{2}+M^{2}}-2 \pi i b_{2}}\right)\right|\right]^{-2} .
$$

\subsection{Manifestly modular invariant one loop free energy}

As a special case of the path integrals in the previous subsection, the partition function of the massive real scalar field $X$ is given by

$$
Z_{X}(M)=\left[e^{2 \pi \tau_{2} \Delta_{0}(M)} \prod_{n}\left(1-e^{-2 \pi \tau_{2}\left|\omega_{n}\right|+2 \pi i \tau_{1} n}\right)\right]^{-1} .
$$

Although GS fermions are not worldsheet fermions, in the path integral formalism they are treated as Grassmann variables only with the different boundary conditions from those of worldsheet fermions. Hence, with a change according to the nature as the Grassmann variables, the direct application of the above result for a complex boson to GS fermions, $S^{a}$, leads to

$$
\begin{aligned}
Z_{S}^{(r, l)}(M) & =e^{2 \pi \tau_{2} \Delta_{b_{1}}(M)} \prod_{n}\left(1-e^{-2 \pi \tau_{2} \sqrt{\left(n+b_{1}\right)^{2}+M^{2}}+2 \pi i \tau_{1}\left(n+b_{1}\right)-2 \pi i b_{2}}\right) \\
& \equiv D_{b_{1}, b_{2}}\left(\tau_{1}, \tau_{2} ; M\right) \quad ; \quad b_{1}=\frac{1-(-1)^{r}}{4}, \quad b_{2}=\frac{1-(-1)^{l}}{4} .
\end{aligned}
$$

This justifies the form of $D_{b_{1}, b_{2}}\left(\tau_{1}, \tau_{2} ; M\right)$ with $\Delta_{b_{1}}(m)$ introduced in a somewhat "ad hoc" way in (4.1). Moreover, resurrecting the full arguments of $G(s)$ as $G_{b_{1}, b_{2}}\left(s ; \tau_{1}, \tau_{2}, M\right)$, one can observe the following modular property of $G(s)$ from the translational symmetry by integer steps in the infinite double summation over $n_{1}$ and $n_{2}$

$$
G_{b_{1}, b_{2}}\left(s ; \tau_{1}, \tau_{2}, M\right)=G_{b_{2},-b_{1}}\left(s ;-\frac{\tau_{1}}{|\tau|^{2}}, \frac{\tau_{2}}{|\tau|^{2}}, M|\tau|\right)=G_{b_{1}, b_{2}+b_{1}}\left(s ; \tau_{1}+1, \tau_{2}, M\right) .
$$

Note that we can always take $0 \leq b_{1,2}<1$ by a suitable translation. These properties are transferred to $G^{\prime}(0)$ which explains the modular property of $D_{b_{1}, b_{2}}(M)$ in (4.5).

After all these, the transverse partition function is given by

$$
\begin{aligned}
Z_{\text {trans }}^{(r, l)}\left(\tau_{1}, \tau_{2}\right) & =\left[Z_{X}\left(\frac{m^{\prime}}{3}\right) Z_{S}^{(r, l)}\left(\frac{m^{\prime}}{3}\right)\right]^{4}\left[Z_{X}\left(\frac{m^{\prime}}{6}\right) Z_{S}^{(r, l)}\left(\frac{m^{\prime}}{6}\right)\right]^{4} \\
& =\left[\frac{D_{b_{1}, b_{2}}\left(\tau_{1}, \tau_{2} ; \frac{m^{\prime}}{3}\right)}{D_{0,0}\left(\tau_{1}, \tau_{2} ; \frac{m^{\prime}}{3}\right)}\right]^{4}\left[\frac{D_{b_{1}, b_{2}}\left(\tau_{1}, \tau_{2} ; \frac{m^{\prime}}{6}\right)}{D_{0,0}\left(\tau_{1}, \tau_{2} ; \frac{m^{\prime}}{6}\right)}\right]^{4} .
\end{aligned}
$$


After plugging (5.32) in (5.13), the final expression of the one loop free energy in the path integral approach is given by

$$
-F=\int_{\mathcal{F}} \frac{d \tau_{1} d \tau_{2}}{2 \tau_{2}} \sum_{r, l \in \mathbf{Z}} \frac{L}{4 \pi^{2} \alpha^{\prime} \tau_{2}} e^{-S_{\beta}(r, l)}\left[\frac{D_{b_{1}, b_{2}}\left(\tau_{1}, \tau_{2} ; \frac{m^{\prime}}{3}\right)}{D_{0,0}\left(\tau_{1}, \tau_{2} ; \frac{m^{\prime}}{3}\right)}\right]^{4}\left[\frac{D_{b_{1}, b_{2}}\left(\tau_{1}, \tau_{2} ; \frac{m^{\prime}}{6}\right)}{D_{0,0}\left(\tau_{1}, \tau_{2} ; \frac{m^{\prime}}{6}\right)}\right]^{4},
$$

where $m^{\prime}$ and $b_{1,2}$ are defined in (5.12) and (5.30), respectively.

The transverse partition function has the following modular property, as can be seen from that of $G(s)$,

$$
Z_{\text {trans }}^{(r, l)}\left(\tau_{1}, \tau_{2}\right)=Z_{\text {trans }}^{(l,-r)}\left(-\frac{\tau_{1}}{|\tau|^{2}}, \frac{\tau_{2}}{|\tau|^{2}}\right)=Z_{\text {trans }}^{(r, l+r)}\left(\tau_{1}+1, \tau_{2}\right)
$$

from which the modular invariance of the one loop free energy (5.33) is manifest.

\subsection{The modular invariance of vacuum amplitude and the flat space limit}

Now we would like to comment on the thermal GS superstrings on flat space. If we take the correct $m$ ( or $M) \rightarrow 0$ limit, we should obtain the same free energy expression as the one obtained in the RNS formalism [2]. The only subtlety in taking the limit arises in the term $Z_{X}(M)$. While $Z_{X}(M) Z_{S}^{(r, l)}(M)=1$ for even $r$ and $l$ and for non-zero $M$, as can be seen from (5.29) and (5.30), $Z_{X}(0) Z_{S}^{(r, l)}(0)=0$ for even $r$ and $l$ due to the fermion zero modes. This shows that the flat space limit is not smooth in the transverse partition function in our formulation. Adopting the correct zero mass limit $Z_{S}^{(r, l)}(0)=0$, for even $r$ and $l,\left[Z_{X}(0)\right]^{8}=V_{8} /\left(4 \pi^{2} \alpha^{\prime} \tau_{2}\right)^{4}|\eta(\tau)|^{16}$ and

$$
D_{0,1 / 2}\left(\tau_{1}, \tau_{2} ; 0\right)=\left|\frac{\theta_{2}(\tau)}{\eta(\tau)}\right|, \quad D_{1 / 2,0}\left(\tau_{1}, \tau_{2} ; 0\right)=\left|\frac{\theta_{4}(\tau)}{\eta(\tau)}\right|, \quad D_{1 / 2,1 / 2}\left(\tau_{1}, \tau_{2} ; 0\right)=\left|\frac{\theta_{3}(\tau)}{\eta(\tau)}\right|
$$

one can see that the one loop free energy becomes

$$
\begin{array}{r}
-F=L V_{8} \int_{\mathcal{F}} \frac{d \tau_{1} d \tau_{2}}{2 \tau_{2}}\left(\frac{1}{4 \pi^{2} \alpha^{\prime} \tau_{2}}\right)^{5}\left|\frac{1}{\eta(\tau)}\right|^{24}\left\{\sum_{\substack{r: \text { even } \\
l: \text { odd }}} e^{-S_{\beta}(r, l)}\left|\theta_{2}(\tau)\right|^{8}\right. \\
\left.+\sum_{\substack{r: \text { odd } \\
l: \text { even }}} e^{-S_{\beta}(r, l)}\left|\theta_{4}(\tau)\right|^{8}+\sum_{\substack{r: \text { odd } \\
l: \text { odd }}} e^{-S_{\beta}(r, l)}\left|\theta_{3}(\tau)\right|^{8}\right\} .
\end{array}
$$

Using the Jacobi identity $\theta_{3}^{4}(\tau)-\theta_{2}^{4}(\tau)-\theta_{4}^{4}(\tau)=0$, this can be put into the form given in [2]

$$
\begin{aligned}
-\frac{F}{V}= & \frac{1}{4} \int_{\mathcal{F}} \frac{d \tau_{1} d \tau_{2}}{2 \tau_{2}}\left(\frac{1}{4 \pi^{2} \alpha^{\prime} \tau_{2}}\right)^{5}\left|\frac{1}{\eta(\tau)}\right|^{24} \sum_{r, l \in \mathbf{Z}} e^{-S_{\beta}(r, l)}\left\{\left(\theta_{2}^{4} \bar{\theta}_{2}^{4}+\theta_{3}^{4} \bar{\theta}_{3}^{4}+\theta_{4}^{4} \bar{\theta}_{4}^{4}\right)\right. \\
& \left.+e^{\pi i(r+l)}\left(\theta_{2}^{4} \bar{\theta}_{4}^{4}+\theta_{4}^{4} \bar{\theta}_{2}^{4}\right)-e^{\pi i r}\left(\theta_{2}^{4} \bar{\theta}_{3}^{4}+\theta_{3}^{4} \bar{\theta}_{2}^{4}\right)-e^{\pi i l}\left(\theta_{3}^{4} \bar{\theta}_{4}^{4}+\theta_{4}^{4} \bar{\theta}_{3}^{4}\right)\right\}
\end{aligned}
$$

where $V=L V_{8}$.

Finally, let us discuss the $(r, l)=(0,0)$ part of the free energy (5.33), which is nothing but the one loop vacuum amplitude. The modular invariance of this amplitude, which is one 
of the consistency check of the given metric as a string theory background, is guaranteed by construction in our path integral approach. In fact, since $m^{\prime}=0$ when $(r, l)=(0,0)$, the one loop vacuum amplitude becomes zero through the correct $m^{\prime}=0$ limit (viz flat space limit). This is in agreement with the fact that the one loop vacuum amplitude of the superstring on the globally supersymmetric background should be zero by the cancellation of the bosonic and fermionic vacuum energies.

\section{Equivalence between operator and path integral methods}

In this section we show the complete agreement between operator and path integral approaches. Apparently, two approaches give the different integration and summation regions in the free energy expression. The free energy (5.33) from the path integral approach is written as the integral over the fundamental region of the torus, $\mathcal{F}$, with the summations over two indices $r$ and $l$, while the one in the operator method is given by the integral over the half strip region,

$$
E:=\left\{\left(\tau_{1}, \tau_{2}\right) ; \quad-\frac{1}{2} \leq \tau_{1} \leq \frac{1}{2}, \quad \tau_{2} \geq 0, \quad\right\},
$$

with the summation over $l$ only. We follow the arguments for the flat space case to show the equivalence of these two forms of the free energy through the resummation along with the change of the integration region.

In the case of type II superstrings on flat space [38, 39], the resummation with the change of the integration region can be summarized as

$$
\sum_{(r, l) \neq(0,0)}^{r, l \in \mathbf{Z}} \int_{\mathcal{F}} \frac{d \tau_{1} d \tau_{2}}{2 \tau_{2}^{2}} e^{-S_{\beta}(r, l)} K_{(r, l)}\left(\tau_{1}, \tau_{2}\right)=\sum_{l \neq 0}^{l \in \mathbf{Z}} \int_{E} \frac{d \tau_{1} d \tau_{2}}{2 \tau_{2}^{2}} e^{-S_{\beta}(0, l)} K_{(0, l)}\left(\tau_{1}, \tau_{2}\right),
$$

where $K_{(r, l)}\left(\tau_{1}, \tau_{2}\right)$ is a function of modular parameter with the modular property

$$
K_{(r, l)}\left(\tau_{1}, \tau_{2}\right)=K_{(l,-r)}\left(-\frac{\tau_{1}}{|\tau|^{2}}, \frac{\tau_{2}}{|\tau|^{2}}\right)=K_{(r, l+r)}\left(\tau_{1}+1, \tau_{2}\right) .
$$

Note that since the transverse partition function $Z_{\text {trans }}^{(r, l)}\left(\tau_{1}, \tau_{2}\right)$ has the same modular property, given in (5.34), as $K_{(r, l)}\left(\tau_{1}, \tau_{2}\right)$, the above resummation with the change of the integration region can be applied to our case as well. Moreover, since $(r, l)=(0,0)$ term has no contribution in the free energy (5.33), it does not have any effect in the resummation with the change of the integration region.

After applying this resummation with the change of the integration region on the free energy (5.33), and by noting that $m^{\prime}$ in (5.12), for $r=0$, becomes $m$ in (3.5), the free energy from the path integral approach (5.33) can be written as

$$
\begin{aligned}
-F=\int_{E} \frac{d \tau_{1} d \tau_{2}}{2 \tau_{2}} \frac{L}{4 \pi^{2} \alpha^{\prime} \tau_{2}}\{ & \sum_{l \neq 0}^{l \in 2 \mathbf{Z}} e^{-\frac{\beta^{2} l^{2}}{4 \pi \alpha^{\prime} \tau_{2}}}+ \\
& \left.\sum_{l}^{l \in 2 \mathbf{Z}+1} e^{-\frac{\beta^{2} l^{2}}{4 \pi \alpha^{\prime} \tau_{2}}}\left[\frac{D_{0,1 / 2}\left(\tau_{1}, \tau_{2} ; \frac{m}{3}\right)}{D_{0,0}\left(\tau_{1}, \tau_{2} ; \frac{m}{3}\right)}\right]^{4}\left[\frac{D_{0,1 / 2}\left(\tau_{1}, \tau_{2} ; \frac{m}{6}\right)}{D_{0,0}\left(\tau_{1}, \tau_{2} ; \frac{m}{6}\right)}\right]^{4}\right\},
\end{aligned}
$$


which is identical with the result from the operator method given in Eq. (4.4).

As we mentioned earlier, the alternative definition of the Hagedorn temperature is the temperature where the thermal winding modes become massless. In order to see this definition also gives the same result, we perform the $\mathcal{S}$-modular transformation,

$$
e^{-S_{\beta}(r, l)} Z_{\text {trans }}^{(r, l)}\left(\tau_{1}, \tau_{2}\right)=e^{-S_{\beta}(l,-r)} Z_{\text {trans }}^{(l,-r)}\left(-\frac{\tau_{1}}{|\tau|^{2}}, \frac{\tau_{2}}{|\tau|^{2}}\right),
$$

which exchanges the winding and momentum modes and do the resummation with the change of integration region. After these computations on the free energy (5.33), it becomes

$$
\begin{aligned}
-F= & \int_{E} \frac{d \tau_{1} d \tau_{2}}{2 \tau_{2}} \frac{L}{4 \pi^{2} \alpha^{\prime} \tau_{2}}\left\{\sum_{r \neq 0}^{r \in 2 \mathbf{Z}} e^{-\frac{\beta^{2} r^{2}}{4 \pi \alpha^{\prime} \tau_{2}}}+\right. \\
& \left.\sum_{r}^{r \in 2 \mathbf{Z}+1} e^{-\frac{\beta^{2} r^{2}}{4 \pi \alpha^{\prime} \tau_{2}}}\left[\frac{D_{1 / 2,0}\left(-\frac{\tau_{1}}{|\tau|^{2}}, \frac{\tau_{2}}{|\tau|^{2}} ; \frac{m}{3}|\tau|\right)}{D_{0,0}\left(-\frac{\tau_{1}}{|\tau|^{2}}, \frac{\tau_{2}}{|\tau|^{2}} ; \frac{m}{3}|\tau|\right)}\right]^{4}\left[\frac{D_{1 / 2,0}\left(-\frac{\tau_{1}}{|\tau|^{2}}, \frac{\tau_{2}}{|\tau|^{2}} ; \frac{m}{6}|\tau|\right)}{D_{0,0}\left(-\frac{\tau_{1}}{|\tau|^{2}}, \frac{\tau_{2}}{|\tau|^{2}} ; \frac{m}{6}|\tau|\right)}\right]^{4}\right\},
\end{aligned}
$$

whose asymptotics is given by (4.7). This shows that the summation index $l$ in the asymptotic expression of one loop free energy (4.7) can be interpreted as the index for winding modes. The lowest winding mode becomes tachyonic at the Hagedorn temperature [2, 7].

We end this section with remarks clarifying subtle points associated with these two approaches. First, we need the renormalization prescription (like Eq. (5.27)) in the path integral approach while it is already done implicitly in the operator method. The normal ordering prescription in the operator approach is a kind of renormalization prescription for free fields, but it is not incorporated in the path integral.

Second, the factor $\tau_{2}$ in Eq. (5.19) was not explicitly included in [33], in contrast to the string theory literatures [31, 32, 5]. Nevertheless, we should have the same physical quantity because it comes from the Lagrangian measure of the coordinate transformation. This can be understood from the fact that the physical quantity we will get after imposing the renormalization condition is independent of this factor (and, of course, the renormalization scale, $\left.\mu_{R}\right)$. This is also obvious from the fact that we can take the new renormalization scale as $\tilde{\mu}=\mu_{R} / \sqrt{\tau_{2}}$ in Eq. (5.19).

Third, the $\zeta$-function method gives us the regularized form of the zero point energy directly, and this gives the identical result with the one from the standard method for the zero point (or Casimir) energy

$$
E_{b}^{\text {Casimir }}(m)=\frac{1}{2} \sum_{n=-\infty}^{\infty} \sqrt{(n+b)^{2}+m^{2}}-\frac{1}{2} \int_{-\infty}^{\infty} d k \sqrt{(k+b)^{2}+m^{2}}
$$

which satisfy the criterion $E_{b}^{\text {Casimir }}(m) \rightarrow 0$ when $m \rightarrow \infty$. Though this $E_{b}^{\text {Casimir }}(m)$ is identical with $\Delta_{b}(m)$, we do not need this regularization as a separate treatment in the operator method or the path integral one because we have already fixed the renormalization prescription in the former one by the normal ordering and in the latter one by the $\zeta$-function with a suitable renormalization condition. 
Finally, since the Hagedorn temperature dependence on the RR flux is entirely given by the difference of bosons and fermions zero point energy as indicated in Eq. (4.8), it does not depend on whether we use the renormalized zero point energy or the physical one ${ }^{5}$.

\section{Acknowledgments}

One of us (J.-D. P.) would like to thank the Yonsei Visiting Research Center (YVRC) for its hospitality, where this work has been completed. The work of S.H. was supported by Korea Research Foundation Grant (KRF-2002-042-C00010).

\section{Appendix}

\section{A. Heuristic derivation of the one loop free energy}

In this appendix, we use the Coleman-Weinberg formula [1, 30] to obtain the one loop free energy, which results in

$$
-\beta F=Z_{T^{2}}(\beta)=-i \int_{\mathcal{F}} \frac{d \tau_{1} d \tau_{2}}{2 \tau_{2}} \sum_{r, l \in \mathbf{Z}} \frac{\beta L}{(2 \pi)^{2}} \int d p^{+} d p^{-} e^{2 \pi \tau_{2} \alpha^{\prime} p^{+} p^{-}} e^{-S_{\beta}(r, l)} Z_{\text {trans }}^{(r, l)}\left(\tau_{1}, \tau_{2}\right) .
$$

Recalling the Wick rotation $p^{0}=i p_{E}^{0}$ and $p^{ \pm}=\left(p^{0} \pm p^{9}\right) / \sqrt{2}$, one can see that this is identical with the previous one loop free energy (5.13) through

$$
\frac{\beta L}{(2 \pi)^{2}} \int d p^{+} d p^{-} e^{2 \pi \tau_{2} \alpha^{\prime} p^{+} p^{-}}=i \frac{\beta L}{4 \pi^{2} \alpha^{\prime} \tau_{2}} .
$$

The transverse partition function, in this approach, is represented as an operator trace [7]

$$
Z_{\text {trans }}^{(r, l)}\left(\tau_{1}, \tau_{2}\right) \equiv \operatorname{tr}_{\text {trans }}\left[(-1)^{(l+1) \mathbf{F}} e^{-2 \pi \tau_{2} \mathcal{H}(r)+2 \pi i \tau_{1} \mathcal{P}}\right]
$$

where $\mathcal{H}(r)$ is defined by the same form as (2.6) while the integer $n$ in $\omega_{n}$ and $\omega_{n}^{\prime}$ is replaced by $n+\frac{1-(-1)^{r}}{4}$.

The naive application to $(r, l)=(0,0)$ mode of the free energy expression (A.1) with the above operator form of the transverse partition function gives

$$
\begin{aligned}
-\beta F & =Z_{T^{2}}(\beta) \\
& =\int_{\mathcal{F}} \frac{d \tau_{1} d \tau_{2}}{2 \tau_{2}} \frac{-i \beta L}{(2 \pi)^{2}} \int d p^{+} d p^{-} e^{2 \pi \tau_{2} \alpha^{\prime} p^{+} p^{-}} \operatorname{tr}_{\text {trans }}\left[(-1)^{\mathbf{F}} e^{-2 \pi \tau_{2} \mathcal{H}+2 \pi i \tau_{1} \mathcal{P}}\right] .
\end{aligned}
$$

After $\beta$ is regarded as another longitudinal length, this is identical with the one loop vacuum amplitude in [22] except for the details in our IIA setting. Apparently, it seems to be divergent, but, we believe, it is the artifact of the light-cone gauge $X^{+} \sim \sigma^{0}$ chosen

\footnotetext{
${ }^{5}$ The previous numerical mismatch $[$, 10 for the Hagedorn temperature in IIB strings on pp-wave comes from the incorrect series expansion for the zero point energy [5] and it has no relation with the formalism or the renormalization.
} 
in the operator formalism. As we showed in the main text, the same quantity without the light-cone gauge fixing in the path integral formalism is zero. Since our background is consistent and globally supersymmetric, the one loop vacuum amplitude should be zero by the cancellation of the bosonic and fermionic vacuum energies. Therefore the above expression of one loop free energy should be understood as the one without $(r, l)=(0,0)$ term in the summation.

\section{B. Useful formulae}

In this section, we derive, following the methods in Ref. [40], the series expansion formula of the function, $\Delta_{b}(m)$, defined by Eq. (4.2) in the more general setting than that in Ref. [10]. Since this expansion can cover the more general twisted boundary conditions, it may be useful for studying the small $\mu$ dependence of the Hagedorn temperature in orbifolded or DLCQ pp-wave case.

Firstly, let us consider the following function

$$
\mathcal{S}(s)_{(b, m)}:=\sum_{n=-\infty}^{\infty} \frac{1}{\left[(n+b)^{2}+m^{2}\right]^{s}}, \quad \operatorname{Re}(s)>\frac{1}{2} .
$$

Note that we can take $0 \leq b<1$ without loss of the generality from the translational symmetry. This function can be represented through the Schwinger's proper time parametrization, $1 / z^{s}=\int_{0}^{\infty} d t t^{s-1} e^{-t z} / \Gamma(s)$, as

$$
\begin{aligned}
\mathcal{S}(s)_{(b, m)} & =\frac{1}{\Gamma(s)} \int_{0}^{\infty} d t t^{s-1} e^{-t m^{2}} \sum_{n=-\infty}^{\infty} e^{-t(n+b)^{2}} \\
& =\frac{\sqrt{\pi}}{\Gamma(s)}\left[\int_{0}^{\infty} d t t^{\left(s-\frac{1}{2}\right)-1} e^{-t m^{2}}+2 \sum_{p=1}^{\infty} \cos (2 \pi b p) \int_{0}^{\infty} d t t^{\left(s-\frac{1}{2}\right)-1} e^{-t m^{2}-\frac{\pi^{2} p^{2}}{t}}\right] \\
& =\sqrt{\pi} m^{1-2 s} \frac{\Gamma\left(s-\frac{1}{2}\right)}{\Gamma(s)}+\frac{4 \pi^{s} m^{\frac{1}{2}-s}}{\Gamma(s)} \sum_{p=1}^{\infty} \frac{\cos (2 \pi b p)}{p^{\frac{1}{2}-s}} K_{\frac{1}{2}-s}(2 \pi m p)
\end{aligned}
$$

where we have used the Poisson resummation formula in the second line and the integral representation of the modified Bessel function $K_{\nu}(z)$ for the second term in the third line

$$
\begin{aligned}
& \sum_{n=-\infty}^{\infty} e^{-t(n+b)^{2}}=\sqrt{\frac{\pi}{t}} \sum_{p=-\infty}^{\infty} e^{-\frac{\pi^{2}}{t} p^{2}+2 \pi i b p}=\sqrt{\frac{\pi}{t}}\left[1+2 \sum_{p=1}^{\infty} e^{-\frac{\pi^{2}}{t} p^{2}} \cos (2 \pi b p)\right], \\
& K_{\nu}(z)=\frac{1}{2}\left(\frac{z}{2}\right)^{\nu} \int_{0}^{\infty} d t t^{-\nu-1} e^{-t-\frac{z^{2}}{4 t}} .
\end{aligned}
$$

Next, note that $\mathcal{S}(s)_{(b, m)}$ can also be expanded as a power series in terms of $m$ as,

$$
\mathcal{S}(s)_{(b, m)}= \begin{cases}\frac{1}{m^{2 s}}+2 \sum_{n=1}^{\infty} \frac{1}{n^{2 s}}\left[1+\frac{m^{2}}{n^{2}}\right]^{-s}, & b=0, \\ \sum_{n=0}^{\infty} \frac{1}{(n+b)^{2 s}}\left[1+\frac{m^{2}}{(n+b)^{2}}\right]^{-s}+\sum_{n=0}^{\infty} \frac{1}{(n+1-b)^{2 s}}\left[1+\frac{m^{2}}{(n+1-b)^{2}}\right]^{-s}, & 0<b<1,\end{cases}
$$




$$
= \begin{cases}\frac{1}{m^{2 s}}+2 \zeta(2 s)-2 s \zeta(2 s+2) m^{2}+2 \sum_{k=2}^{\infty} \frac{(-1)^{k} \Gamma(k+s)}{\Gamma(s) \Gamma(k+1)} \zeta(2 k+2 s) m^{2 k}, \quad b=0, \\ \zeta(2 s, b)+\zeta(2 s, 1-b)-s[\zeta(2 s+2, b)+\zeta(2 s+2,1-b)] m^{2} \\ \quad+\sum_{k=2}^{\infty} \frac{(-1)^{k} \Gamma(k+s)}{\Gamma(s) \Gamma(k+1)}[\zeta(2 k+2 s, a)+\zeta(2 k+2 s, 1-a)] m^{2 k}, & 0<b<1,\end{cases}
$$

where $\zeta(s)$ and $\zeta(s, q)$ are the (Riemann) zeta function and the generalized (or RiemannHurwitz) zeta function, respectively, defined as

$$
\zeta(s):=\sum_{n=1}^{\infty} \frac{1}{n^{s}}, \quad \zeta(s, q):=\sum_{n=0}^{\infty} \frac{1}{(n+q)^{s}}, \quad q \neq 0,-1,-2, \cdots .
$$

Some useful formulae for them are

$$
\begin{aligned}
& \zeta(s)=\zeta(s, 1), \quad \zeta\left(s, \frac{1}{2}\right)=\left(2^{s}-1\right) \zeta(s), \\
& \zeta(-1, q)=\frac{1}{24}-\frac{1}{8}(2 q-1)^{2}, \quad \zeta(s, q)=\frac{1}{s-1}-\psi(q)+\mathcal{O}(s-1)
\end{aligned}
$$

where $\psi(q):=\frac{d}{d q} \ln \Gamma(q)$ with $\psi(1)=-\gamma_{E}=-0.5772 \ldots$ and $\psi(1 / 2)=-\gamma_{E}-2 \ln 2$. Here, $\gamma_{E}$ is the Euler constant.

Now, we identify Eq.(B.2) with Eq.(B.3) for sufficiently large and positive $s$-values, and perform the analytic continuation to the entire $s$-complex plane. Then, we can see that the function, $\Delta_{b}(m)$, can be represented as

$$
2 \Delta_{b}(m) \equiv-\frac{2 m}{\pi} \sum_{p=1}^{\infty} \frac{\cos (2 \pi b p)}{p} K_{1}(2 \pi m p)=\lim _{s \rightarrow-\frac{1}{2}}\left[\mathcal{S}(s)_{(b, m)}-\sqrt{\pi} m^{1-2 s} \frac{\Gamma\left(s-\frac{1}{2}\right)}{\Gamma(s)}\right] .
$$

When we take $s \rightarrow-\frac{1}{2}$ in the above, the would-be pole part $1 /\left(s+\frac{1}{2}\right)$ in the series form of $\mathcal{S}(s)_{(b, m)}$, which comes from $\zeta(2 s+2)$ or $\zeta(2 s+2, b)+\zeta(2 s+2,1-b)$ at $m^{2}$ order in Eq. (B.3), cancels out exactly with the one from the Laurent expansion

$$
\sqrt{\pi} m^{1-2 s} \frac{\Gamma\left(s-\frac{1}{2}\right)}{\Gamma(s)}=\frac{1}{2} m^{2}\left[\frac{1}{s+\frac{1}{2}}-\ln \frac{m^{2}}{4}+1\right]+\mathcal{O}\left(s+\frac{1}{2}\right) .
$$

We emphasize that the above procedure through Eq. (B.6) does not involve the divergent expression which needs a regularization but just gives us a mathematical relation by the analytic continuation ${ }^{6}$.

\footnotetext{
${ }^{6}$ Note that Eq. ( $\left.\sqrt{\text { B.1 }}\right)$ is used just as an intermediate step rather than the aim contrary to Ref. 40. We might, of course, take this directly as a regularized definition for the zero point energy like in Ref. [40]. But this requires the physical consideration leading the renormalization condition. Therefore, our logic is different from Ref. 40] .
} 
With Eq.s (B.3), (B.5), we can finally get the power series expansion for $\Delta_{b}(m)$ in terms of $m$ as,

$$
\Delta_{b}(m)=\left\{\begin{array}{rlrl}
-\frac{1}{12} & +\frac{1}{2} m+\frac{1}{4} m^{2}\left[\ln \frac{m^{2}}{4}+2 \gamma_{E}-1\right]+\sum_{k=2}^{\infty} \frac{(-1)^{k} \Gamma\left(k-\frac{1}{2}\right)}{\Gamma\left(-\frac{1}{2}\right) \Gamma(k+1)} \zeta(2 k-1) m^{2 k}, \quad b=0 \\
\frac{1}{24} & -\frac{1}{8}(2 b-1)^{2}+\frac{1}{4} m^{2}\left[\ln \frac{m^{2}}{4}-\psi(b)-\psi(1-b)-1\right] \\
& +\sum_{k=2}^{\infty} \frac{(-1)^{k} \Gamma\left(k-\frac{1}{2}\right)}{\Gamma\left(-\frac{1}{2}\right) \Gamma(k+1)} \frac{1}{2}[\zeta(2 k-1, b)+\zeta(2 k-1,1-b)] m^{2 k}, & 0<b<1 .
\end{array}\right.
$$

The special cases $b=0$ and $b=1 / 2$ give us

$$
\Delta_{1 / 2}(m)-\Delta_{0}(m)=\frac{1}{8}-\frac{1}{2} m+m^{2} \ln 2+\sum_{k=2}^{\infty} \frac{(-1)^{k} \Gamma\left(k-\frac{1}{2}\right)}{\Gamma\left(-\frac{1}{2}\right) \Gamma(k+1)}\left(2^{2 k-1}-2\right) \zeta(2 k-1) m^{2 k},
$$

which is the identical expression with the one in Ref. [10, 33. 


\section{References}

[1] J. Polchinski, "String Theory. Vol. 1, 2", Cambridge, Uk: Univ. Pr. (1998).

[2] J. J. Atick and E. Witten, Nucl. Phys. B 310 (1988) 291.

[3] R. Hagedorn, Nuovo Cim. Suppl. 3 (1965) 147.

[4] K. Huang and S. Weinberg, Phys. Rev. Lett. 25, 895 (1970).

[5] L. A. Pando Zayas and D. Vaman, [arXiv:hep-th/0208066].

[6] B. R. Greene, K. Schalm and G. Shiu, Nucl. Phys. B 652 (2003) 105 [arXiv:hep-th/0208163].

[7] Y. Sugawara, Nucl. Phys. B 650 (2003) 75 [arXiv:hep-th/0209145].

[8] R. C. Brower, D. A. Lowe and C. I. Tan, Nucl. Phys. B 652 (2003) 127 [arXiv:hep-th/0211201].

[9] Y. Sugawara, [arXiv:hep-th/0301035].

[10] G. Grignani, M. Orselli, G. W. Semenoff and D. Trancanelli, [arXiv:hep-th/0301186].

[11] M. Blau, J. Figueroa-O'Farrill, C. Hall and G. Papadopoulus, JHEP 0201 (2001) 047, [arXiv:hep-th/0110242].

[12] R. R. Metsaev, Nucl. Phys. B 625 (2002) 70 [arXiv:hep-th/0112044]; R. R. Metsaev and A. A. Tseytlin, Phys. Rev. D 65 (2002) 126004 [arXiv:hep-th/0202109].

[13] D. Berenstein, J. M. Maldacena and H. Nastase, JHEP 0204 (2002) 013 [arXiv:hep-th/0202021].

[14] S. Hyun and H. Shin, JHEP 0210 (2002) 070 [arXiv:hep-th/0208074].

[15] J. Figueroa-O'Farrill, G. Papadopoulos, JHEP 0108 (2001) 036, [arXiv:hep-th/0105308].

[16] S. Frautschi, Phys. Rev. D 3 (1971) 2821.

[17] R. D. Carlitz, Phys. Rev. D 5 (1972) 3231.

[18] M. J. Bowick and L. C. Wijewardhana, Phys. Rev. Lett. 54 (1985) 2485.

[19] M. J. Bowick and S. B. Giddings, Nucl. Phys. B 325 (1989) 631.

[20] N. Deo, S. Jain and C. I. Tan, Phys. Lett. B 220 (1989) 125; N. Deo, S. Jain and C. I. Tan, Phys. Rev. D 40 (1989) 2626; N. Deo, S. Jain, O. Narayan and C. I. Tan, Phys. Rev. D 45 (1992) 3641.

[21] S. Hyun and H. Shin, Nucl. Phys. B 654 (2003) 114 [arXiv:hep-th/0210158].

[22] T. Takayanagi, JHEP 0212 (2002) 022 [arXiv:hep-th/0206010].

[23] O. Bergman, M. R. Gaberdiel and M. B. Green, JHEP 0303 (2003) 002 [arXiv:hep-th/0205183].

[24] B. Sathiapalan, Phys. Rev. D 35 (1987) 3277.

[25] Y. I. Kogan, JETP Lett. 45 (1987) 709 [Pisma Zh. Eksp. Teor. Fiz. 45 (1987) 556].

[26] J. G. Russo and A. A. Tseytlin, JHEP 0204 (2002) 021 [arXiv:hep-th/0202179].

[27] S. Carlip, Phys. Lett. B 186 (1987) 141; Nucl. Phys. B 284 (1987) 365. 
[28] R. Kallosh and A. Y. Morozov, Int. J. Mod. Phys. A 3 (1988) 1943 [Sov. Phys. JETP 67 (1988 ZETFA,94,42-56.1988) 1540].

[29] A. B. Hammou, JHEP 0211 (2002) 028 [arXiv:hep-th/0209265].

[30] J. Polchinski, Commun. Math. Phys. 104 (1986) 37.

[31] E. Alvarez, Nucl. Phys. B 269 (1986) 596.

[32] E. Alvarez and M. A. Osorio, Phys. Rev. D 36 (1987) 1175.

[33] H. Saleur and C. Itzykson, "Two-Dimensional Field Theories Close to Criticality", J. Statist. Phys. 48 (1987) 449.

[34] J. C. Collins, "Renormalization", Cambridge, Uk: Univ. Pr. (1984) 380p.

[35] M. Bordag, U. Mohideen and V. M. Mostepanenko, Phys. Rept. 353 (2001) 1 [arXiv:quant-ph/0106045].

[36] E. M. Santangelo, Theor. Math. Phys. 131 (2002) 527 [Teor. Mat. Fiz. 131 (2002) 98] [arXiv:hep-th/0104025].

[37] K. A. Milton, [arXiv:hep-th/9901011].

[38] K. H. O'Brien and C. I. Tan, Phys. Rev. D 36 (1987) 1184.

[39] B. McClain and B. D. Roth, Commun. Math. Phys. 111 (1987) 539.

[40] J. Ambjorn and S. Wolfram, Annals Phys. 147 (1983) 1. 